Canadian

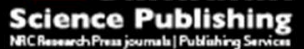

Canadian Journal of Civil Engineering Revue canadienne de génie civil

Myopic Choice or Rational Decision Making? An Investigation into Mode Choice Preference Structures in Competitive Modal Arrangements in a Multimodal Urban Area, the City of Toronto

\begin{tabular}{|r|l|}
\hline Journal: & Canadian Journal of Civil Engineering \\
\hline Manuscript ID & cjce-2015-0336.R1 \\
\hline Manuscript Type: & Article \\
\hline Date Submitted by the Author: & 09-Feb-2016 \\
\hline Complete List of Authors: & $\begin{array}{l}\text { Mahmoud, Mohammed Salah; University of Toronto, Civil Engineering } \\
\text { Weiss, Adam; University of Toronto, Civil Engineering } \\
\text { Nurul Habib, Khandker; University of Toronto, }\end{array}$ \\
\hline Keyword: & Myopic choice, rational decision making, mode choice, bike infrastructure \\
\hline
\end{tabular}




\title{
Myopic Choice or Rational Decision Making? An Investigation into Mode Choice Preference Structures in Competitive Modal Arrangements in a Multimodal Urban Area, the City of Toronto
}

\author{
Mohamed Salah Mahmoud, PhD. \\ Post Doctoral Fellow \\ Department of Civil Engineering \\ University of Toronto
}

35 St. George Street, Toronto, Ontario, M5S 1A4, Canada

Tel: 416-946-8299; Email: mohamed.mahmoud@utoronto.ca

\author{
Adam Weiss, MASc. \\ Ph.D. Candidate \\ Department of Civil Engineering \\ University of Toronto \\ 35 St. George Street, Toronto, Ontario, M5S 1A4, Canada \\ Tel: 416-946-8299; Email: adam.weiss@utoronto.ca
Khandker Nurul Habib, Ph.D ${ }^{1}$., P.Eng.
Associate Professor
Department of Civil Engineering
University of Toronto \\ 35 St. George Street, Toronto, Ontario, M5S 1A4, Canada \\ Tel: 416-946-8027; Email: Khandker.nurulhabib@utoronto.ca
}

\footnotetext{
${ }^{1}$ Corresponding Author. Email: Khandker.nurulhabib@utoronto.ca
} 


\title{
Myopic Choice or Rational Decision Making? An Investigation into Mode Choice Preference Structures in Competitive Modal Arrangements in a Multimodal Urban Area, the City of Toronto
}

\begin{abstract}
This paper presents an investigation into the preference structure of commuting mode choice in dense urban areas. The paper aims to investigate the phenomenon of myopic choice and extends the phenomenon to the concept of modal culture. Using a household travel diary survey from the greater Toronto and Hamilton Area (GTHA), an empirical discrete choice model was estimated. This model was used to provide general comments on the commuting and dependent behaviour of the sample, with a particular focus on the factors that influence bicycling captivation and culture. The model was then used for a hypothetical policy scenario analysis, which found that an investment in biking infrastructure had the capacity to increase bicycling mode share by nearly $50 \%$. Based on this result, this paper recommends further investigation into both data collection for more comprehensive empirical model development and investigation into the policy applicability of the proposed model structure.
\end{abstract}

Keywords: Myopic choice, rational decision making, mode choice, bike infrastructure 


\section{Introduction}

Urban centres are often both central business districts (CBD) and residential hubs, while the land surrounding the urban centre is typically residential. This urban form leads to a large number of internal trips within the urban centre as well as travel to and from the center from the surrounding municipalities. In particular, during peak commuting periods, urban centers are highly congested due to the existing travel demand from the residents within the centre and those commuting into the CBD from residential municipalities outside of the urban centre. There is significant interest in understanding the travel patterns of those who reside within these urban regions as they likely have more options in terms of travel modes available to them, relative to those travelling into the urban centre from the surrounding municipalities. In particular, individuals who reside and work in the same urban regions will often be able to walk or bicycle to work due to the lower travel distance between their origin and destination. Despite this, within the North American context, the market for non-motorized utilitarian travel is largely untapped, with historical bicycle use being predominantly recreational (Dill and Carr 2003). Understanding the decision-making process for mode choice of this segment of the urban population will allow policy makers interested in improving bicycle modal share to target their policies at these groups effectively.

Gaining insight into the factors that incentivizes and constrains modal choice, and in particular, non motorized travel choices for commuting within dense urban regions is essential for shaping policies and practices to encourage the use of non-motorized travel modes for work based trips. The work of Buehler and Pucher (2012) explicitly indicates 
that bicycling infrastructure plays a large role in shaping the propensity for bicycle travel for commuting. Likewise, their analysis of the impact of factors such as temperature and precipitation found an expected decrease in total number of bicycle trips based on the average number of extreme heat days and annual precipitation values, whereas subzero temperatures had limited impact on bicycling trip generation. This finding is in agreement with previous research that found that commuters in major Canadian cites are approximately three times more likely to use their bikes to get to work relative to commuters in American cities (Pucher and Buehler 2006), even though the Canadian cities are more prone to subzero temperatures. Higher urban densities, safer bike conditions and mixed-use development within Canadian cities all contribute to explaining this finding. The work done to understand utilitarian bicycling behaviour provides an aggregate understanding of biking travel patterns in terms of the factors that influence bicycling behaviour for commuting. There has also been some investigation into the disaggregate understanding of individual bicycling behaviour. The work presented in (Habib et al. 2014) provides an empirical discrete choice model that incorporates latent perceptions of bikability and bicycling safety as factors, which influence the choice to bicycle for both utilitarian and recreational purposes.

The definition of modal availability or the omission of specific modes from individuals' choice sets greatly affects the accuracy of studying their mode choice behaviour (Tardiff 1976; Stopher 1980). One case of alternative omission is the dependent or myopic case, whereby the decision maker rejects all alternatives but one, thereby guaranteeing the selection of a single alternative within the universal choice set. In many choice-modelling 
applications, some or all aspects of this dependency are often ignored completely. Typical choice models using the multinomial logit formulation often apply rules to define modal availability (i.e. if the decision maker does not have a driver's license, then driving is not an available alternative to them), thereby capturing what has been previously defined as "forced captivity" (Jacques, Manaugh, and El-Geneidy 2013). This form of forced dependency can be determined using logical rules and is, therefore, relatively easy to encapsulate within most model specifications. Conversely, individuals may have no desire to consider some of the alternatives that may be readily available to them and, therefore, are implicitly dependent. This implicit dependency may be a result of individuals' preconceptions of specific alternatives. For instance, perceived higher mobility with a car or viewing bicycling to work as an efficient way exercise may implicitly make a commuter irrationally attracted to one mode over another. Therefore, even if a mode may become more attractive from a level of service perspective, the decision maker will not consider the rational tradeoff between modes. Unfortunately it is difficult to directly capture such implicit decisions regarding which alternatives to consider within individuals' choice sets. Therefore, modellers often treat the perception of modal dependency as a myopic choice. Despite these modelling challenges, understanding how myopic dependency impacts commuting mode choice is highly relevant for policy and decision makers. This stems from the concept that dependent users will inherently not consider alternative modes, even if they become more attractive from a level of service perspective. This has implications for determining policies to encourage mode-switching behaviour, as discussed in Habib and Weiss (2014). 
Given that dependency on a specific mode is typically defined by personal factors or mode perceptions, behavioural models that capture myopic dependency can be used as a method for understanding the impact of the built environment (as a proxy for these perceptions) on travel mode culture. The work of Aldred and Jungnickel (2014) accesses the implications of culture within transportation policy on bicycling in the United Kingdom. They define culture as "everyday behaviour" where an individual's choice is made based on social norms without a conscientious consideration of their options. They further defined culture as being complementarily defined by both a "behavioral habit", much like that discussed by Tudela et al (2011), that is formed over time due to repeated behaviour, and a "reaction to the environment" whereby the decision-maker has particular options enabled or blocked based on her reaction to her personal situation. When compared to the concepts and discussions of myopic choice, obvious parallels between the reactionary component of culture and myopic choice to some modes can be found. Specifically, the concept of myopic choice on an aggregate level is analogous to capturing modal culture within a region. As such, this paper provides a preliminary investigation into the application of advanced econometric discrete choice modeling structures as a proxy for examining this portion of modal culture. Specifically, this paper presents and empirical application of the parameterized logit captivity (PLC) model originally proposed by Ben-Akiva and Swait $(1984 ; 1987 b)$. By examining the myopic choice or dependence behaviour outlined below, it is possible to obtain deeper insight into the modal culture of a region relative to the understanding gained from conventional models or mode split analysis. 
To explore this application of the model, the remainder of this paper will outline the existing literature on alternative myopic choice modeling with a focus on the parameterized logit captivity (PLC) model. Then, the paper presents an empirical model using the PLC formulation for the city of Toronto. The model is then used as the basis for a policy analysis scenario, where the implications of infrastructure investment on cycling culture and changing biking modal captivation investigated.

\section{Literature review}

Modal dependence and choice set generation are concepts with significant interest and development within the field of discrete choice modelling. Manski (1977) introduced the problem of choice set generation for decision-makers; however, he provides limited discussion on the concept of myopic modal dependence. Instead, Manski's discussion postulates that through sufficient details and personalized information regarding the decision-maker and associated choice scenario, the myopic choice set can be indirectly inferred. Such information is often not available within collected travel behaviour data. Therefore an alternative method to capture choice set generation, and by extension, myopic choice is required to fill this gap in the literature.

Before a formal modelling approach to address the issue of myopic choice is presented, it is first pertinent to review the work of Stopher (1980) who proves that failure to capture myopic consideration within a choice model structure results in choice model misspecification. Further, the work of Swait and Ben-Akiva (1986) highlights the need 
for understanding the effect of myopic modal dependence on modal availabilities and choices in regions with rapidly changing social and economic structures. The Dogit model was the first model structure that addressed the myopic reliance concept in the context of modal choice (Gaundry and Dagenais 1979). The Dogit model was originally formulated as an alternative to the logit structure in order to relax the IIA assumption inherent within the multinomial logit (MNL) formulation. Swait and Ben-Akiva (1987a; 1987b) further interpreted the Dogit model as a model capable of predicting alternative reliance. The Dogit model consists of two portions, an irrational dependent portion and a rational portion. Swait and Ben-Akiva further expanded the constant-only "myopic" odds parameter present in the irrational portion of the original Dogit model, into a parameterized function. The analysis performed by Bordley(1990) suggests that the original form of the Dogit model and the parameterized version are still valid for predicting choice behaviour under incomplete dependency conditions. This finding relaxes the concerns of prior applications of the model, in which decision contexts where complete alternative reliance is not guaranteed were considered.

Although the first attempts to use the Dogit and Parameterized Logit Captivity (PLC) models proved to have significant improvements over corresponding MNL models estimated from the same data (Gaudry 1980; Swait and Ben-Akiva 1986), limited applications of these models have been reported after a few initial attempts. Both the Dogit and PLC models have closed forms of the likelihood functions making the estimation process relatively straightforward and comparable to the standard MNL model. While no formal commercial software has been developed with the explicit 
capacity to estimate the Dogit and PLC models, more general-purpose software (such as R, GAUSS, and STATA) is capable of estimating these models using traditional maximum likelihood estimation techniques.

While there have been relatively few recent applications of the Dogit and PLC models, there are a few notable attempts to apply these models to different contexts. McCarthy (1997) applied the logit captivity model to intercity mode choice, and found the captivity model structure outperformed a model structure that did not consider dependency. Chu (2009) utilized the Dogit Generalized Extreme Value (DOGEV), originally proposed by Fry and Harris (2005) to model commuters' departure time choice. The DOGEV model structure replaces the rational conditional choice portion of the Dogit model structure with a GEV model structure. Other applications by Chu include examining destination location choice using a form of the Dogit model as a means to improve networkmodelling capacity (2010). Recently, the work of Habib and Weiss (2014) presents a version of the PLC estimated with three repeated cross sectional datasets for commuting mode choice. The scale parameter was parameterized within the rational portion of the choice model to account for heterogeneity across the different panel datasets as well as spatial variability. Consistent with other applications of the Dogit and PLC models, the estimated model outperformed the more traditional MNL formulation.

This paper continues to examine the application of the PLC model formulation to study commuting mode choice behaviour within the urban population segment, which is less likely to be forcefully reliant on a certain mode. This application aims to improve the 
understanding of the choice set generation and myopic choice or captivation to some modes over others. The study focuses on understanding bicycling captivation, and by extension bicycling culture for this population segment. The next section of the paper will outline the basic PLC model formulation then a detailed discussion of the study area and empirical model results are presented.

\section{Econometric model}

The PLC model structure presented below is based on the same model structure originally proposed by Gaudrey and Dagenais (1979). The model operates under the assumption that the decision-maker is either rational and, therefore, will select from his or her full choice set, or dependent and as such will automatically select a single alternative under all circumstances, irrespective of the level of service values of other options. It should also be noted that the rational decision maker will have infeasible options removed from their full choice set using a rule based approach as outlined above. Based on this structure, it is possible to define the probability of selecting a mode "m" $\left(P_{m}\right)$ as:

$$
\begin{aligned}
& P_{m}=P_{\text {captive }-m}+P_{m \mid \text { rational }} \times P_{\text {rational }} \\
& \text { where } P_{\text {rational }}=\left(1-\sum_{m^{\prime}} P_{\text {captive }-m^{\prime}}\right)
\end{aligned}
$$


Here $P_{\text {captive }-m}$ refers to the probability of being reliant to mode $\mathrm{m}$ and $P_{m \mid \text { rational }}$ refers to the probability of selecting mode $\mathrm{m}$ given that the decision-maker is choosing from his/her full choice set. The Dogit model can be formulated as:

$$
P_{m}=\frac{U_{m}}{1+\sum_{m^{\prime}} U_{m^{\prime}}}+\frac{1}{1+\sum_{m^{\prime}} U_{m^{\prime}}} P_{m \mid \text { rational }}
$$

Where $U_{m}$ is the myopic odds parameter, specific for alternative m. The work of Swait and Ben-Akiva (1987a; 1987b), further expands on this structure by parameterizing the myopic odds parameter as a linear in parameter function, $D_{m}$, using the following formulation:

$$
P_{m}=\frac{\exp \left(D_{m}\right)}{1+\sum_{m^{\prime}} \exp \left(D_{m^{\prime}}\right)}+\frac{1}{1+\sum_{m^{\prime}} \exp \left(D_{m^{\prime}}\right)} P_{m \mid \text { rational }}
$$

Where $D_{m}$ is a linear in parameter equation of alternative specific or decision maker socioeconomic variables $y$ and corresponding parameter coefficients $\alpha$ with an alternative specific constant, $\delta_{m}$ :

$$
D_{m}=\delta_{m}+\sum \alpha y
$$

The use of socio economic or land use variables for the dependent portion of the model stems from the probability of dependence on a given mode being based more on external or internal values rather than comparisons of level of service values. Alternatively, in the 
constant-only model and the PLC model, the $P_{m \mid \text { rational }}$ term is the well-known multinomial logit model structure, which can be expressed as follows:

$$
\begin{gathered}
P_{m \mid \text { rational }}=\frac{\exp \left(\mu V_{m}\right)}{\sum_{m^{\prime}} \exp \left(\mu V_{m^{\prime}}\right)} \\
V_{m}=A S C_{m}+\sum \beta x
\end{gathered}
$$

Where $V_{m}$ is a linear in parameter function of observed level of service values, $\mathrm{x}$ and the $\beta$ vector represents the corresponding coefficients, $\mu$ is the scale parameter, and $A S C_{m}$ is an alternative specific constant. In the rational portion of the model, the decision maker is assumed to be making a tradeoff between the different alternatives based on his/her attributes, namely, level of service values. Typical applications of the PLC or DOGIT model in the past have assumed constant scale parameter. However, the work of Habib and Weiss (2014) showed that the model is able to account for heterogeneity across the sample population both spatially and temporally through a parameterization of the scale parameter. While the model presented here attempted to parameterize the scale parameter using spatial variables, no spatial variables were found to be significant and thus the scale parameter was fixed to 1 . This can likely be explained by the much smaller study area used for this analysis, relative to the earlier work of Habib and Weiss, resulting in much more homogeneous spatial characteristics. The final model will be expressed based on the equation below: 


$$
P_{m}=\frac{\exp \left(D_{m}\right)}{1+\sum_{m} \exp \left(D_{m}\right)}+\frac{1}{1+\sum_{m} \exp \left(D_{m}\right)} \frac{\exp \left(V_{m}\right)}{\sum_{m} \exp \left(V_{m}\right)}
$$

The application of this model discussed below was estimated using code written for GAUSS, using the MAXLIK procedure for maximum likelihood estimation (Aptech 2014 ). As the likelihood function is highly non-linear, it is important to have initial parameter assumptions are essential for optimal parameter estimation, as the large number of local maxima or apparent flat surfaces within the likelihood function may result in parameter estimates that do not maximize the likelihood. More conventional models were tested first to provide rough approximations for the parameter estimates and particular care was put into assuring overall goodness of fit of each parameter.

\section{Study area and data description}

The City of Toronto (Toronto) is located in the heart of the Greater Toronto Area (GTA), Canada's largest metropolitan area. As of 2011, the reported population was over 2.6 million with a projected growth to 3 million by 2031 (Statistics Canada 2012). The Toronto Transit Commission (TTC), the local transit service, provides extensive network coverage across the city, which results in a transit modal share of $54 \%$ of commuting trips (Data Management Group 2013). In addition, the city is promoting active modes by improving and adding bicycle and walk-friendly infrastructure (Metrolinx 2008). The modal shares of bicycling and walking in Toronto for commuting trips are $5 \%$ and $7 \%$, respectively (Data Management Group 2013). Within the North American context, these modal shares for non-motorized travel are relatively high making the city a perfect case 
study for understanding commuters' mode choice behaviour within the context of nonmotorized travel.

Data from the 2011-2012 Transportation Tomorrow Survey (TTS), a trip-based household survey conducted every five years in the Greater Toronto and Hamilton Area (GTHA) among 5\% of its population, is used in this paper (Data Management Group 2013). A subset of 8,215 records, containing commuting trips for those working and living in Toronto, was used for estimating and validating the empirical models. The data provides information on the trips as well as the socioeconomic and demographics of the trip makers. Five modes are considered in the analysis, namely, auto driver, auto passenger, transit with walk access, biking and walking. Figure 1 shows the mode shares of the five modes in the data set. Figure 2 shows the TTC subway network and a spatial kernel density of the percent of bike infrastructure covering individuals' routes based on trip origins. The map shows that trips that originate from (or are close to) the city centre (Planning District 1) have higher percentages of bicycle infrastructure length. In addition, hourly weather data for 2011/2012, provided by Environment Canada, including temperature, snow on ground, wind speed, precipitation and relative humidity was matched to each trip record.

Level of service attributes, consisting of travel times and travel costs for auto driver, auto passenger and transit modes were generated using EMME4 traffic assignment software and a 2012 scenario of the Greater Toronto Area (GTA). Conversely, travel times for bicycle and walk modes were generated based on the suggested bicycle/walk routes provided by Google Maps ${ }^{\circledR}$ using its Application Programming Interface (API). Further 
spatial analysis was conducted in ESRI ArcMap ${ }^{\circledR} 10.1$ to generate other metrics such as the percent of bicycle infrastructure length compared to the total trip length and number of intersections with major roads.

\section{Empirical model}

Table 1 shows results of the empirical model. The empirical model was estimated using 6,566 trip records which represented $80 \%$ of the available sample size. The remaining $20 \%$ was used for model validation. A total of 27 parameters were estimated. The reported parameters were found to be statistically significant ( $t$-statistics higher than 1.96) with the expected signs. The model showed a relatively high goodness of fit compared to the constant-only model with a reported Rho-squared value of 0.22.

It is also worth mentioning that a corresponding MNL model was estimated using the same dataset. Both models showed similar Rho-squared values, which indicates that the PLC model fits the data as well as the MNL model while providing stronger behavioural insights in the context of mode culture and dependence. In addition, the likelihood ratio test was performed with a test statistics value of 3755.15 indicating that the full model fits the data significantly better than the constant-only model. In addition, the model was validated against the retained $20 \%$ trip records. The "percent correctly predicted" analysis, as another goodness-of-fit statistics (Train 2009), showed that the model performed at a relatively high prediction accuracy of $70 \%$. Further analysis of the model outputs examined the predicted dependent and total modal shares. Based on this, both transit and auto passenger modes were more competitive based on the specified 
systematic utilities, whereas the other alternatives were less competitive, and, therefore, their modal shares were more explained by dependent markets. From a policy perspective, improving factors that influence modal reliance, as defined within the model specification, would have a greater impact than improving the level of service values for these modes. Furthermore, the almost exclusive explanation of biking, walking and driving mode share from the non rational myopic choice suggested a strong presence of modal cultures for these three travel modes within the city of Toronto.

Figures 3 and 4 show heat maps of different levels of auto driver and bicycle reliance probabilities based on the trip origins. The map revealed interesting findings; the probability of auto driver dependence is higher around the city peripheral and decreased as it comes closer to the subway lines or the city centre. The spatial distributions of bicycle reliance probabilities show the concentration of higher probabilities at the city centre. By comparing Figures 2 and 4, the relationship between the concentration of trips with higher probability of bicycle reliance and bike infrastructure was clearly evident. The probability of dependence/ myopic choice of any mode is strongly correlated with the built environment.

In order to capture the spatial heterogeneity across the sample, the authors attempted to parameterize the scale parameter of the rational component of the probability equation. While a number of different spatial variables were tested, it was found that no spatial variables were significant. While not ideal, the lack of significance of the spatial parameters can likely be explained by the constrained study area of just the city of 
Toronto proper and none of the surrounding suburban municipalities. Ideally, economic variables such as income would be included in the scale parameter; however, due to limitations with the data set this was not feasible to test.

Typical levels of service attributes (travel times and cost/fare) were included in the systematic utility/rational choice component of the model as these factors are used to make a rational tradeoff between alternatives. Results showed consistency with previous research findings in that higher travel time and cost were deterrents to modal selection. On the other hand, socioeconomic and demographic attributes and mode-specific levels of service attributes were added to the parameterized myopic odds functions. These factors are more likely to push an individual towards considering one alternative over another. An increase in the number of vehicles per household and the availability of free parking at work location resulted in an increase in the probability of the auto driver reliance. Similarly, individuals with higher number of vehicles compared to the number of persons per household were less likely to only consider transit as his or her travel mode. On the other hand, possessing a transit pass increased the probability of transit dependence. It was also found that males were less likely to be reliant on the auto passenger mode.

More focus was given to active modes, especially bicycling, as an effort to understand some of the factors influencing bicycling culture within the city. Individuals who were 60 years and older were less likely to be reliant on bicycling or walking for their commuting mode choice. On the other hand, individuals who commuted during off-peak 
periods to work location within the city centre (planning district 1) had higher probability of bicycle dependence. Similarly, individuals who lived and worked in the city centre (as a proxy for short distance commuting travel and considering the walk-friendly neighbourhood of Planning District 1) were more likely to be reliant on the walk to work mode. The built environment, and in particular the number of intersections and the presence of dedicated infrastructure also had an impact on perceptions of biking for commuting. These built environment factors have strong implications to the perception of safety while biking, as dedicated, separated bike lanes and fewer intersections should reduce the likelihood of being in a cycling accident. Males who had more than $50 \%$ of their total travelled distance covered by bicycle infrastructure (bicycle lanes) were more likely to consider bicycling as their only mode of commute. As the number of intersections with major roads increases, this probability decreases, implying that cyclists prefer continuous travel routes with little to no interruption caused by waiting at traffic lights as well as the increased safety associated with not having to negotiate mixed traffic. In terms of the weather effect, individuals were more likely to be myopic towards the bicycle mode if the temperature was higher than $10^{\circ} \mathrm{C}$, if the temperature fell below this threshold, an individual's myopic choice of bicycling fell. While the myopic choice of bicycling might decrease under these conditions, it should be noted that the individual might still select biking through a rational choice. However, he or she might also consider other modal options for commuting under these conditions. Conceptually, this behaviour makes sense, as a typical myopic biking commuter who faced adverse weather conditions might debate his or her modal choice and perform a rational trade off. For example, if it is cold out, a decision-maker might be more inclined to consider other modal alternatives, 
as he or she may change their reliance from season to season. It should also be noted that the maximum reported temperature for the sampled trips was $25^{\circ} \mathrm{C}$. The minimum temperature was $-11^{\circ} \mathrm{C}$ and the average was $8.2^{\circ} \mathrm{C}$. Given that during the winter months in Toronto, it is not unusual to experience temperatures well below $-20^{\circ} \mathrm{C}$ and during the summer the temperatures can be well over $30^{\circ} \mathrm{C}$ this sample gives only a partial view of the bicycle commuting behaviour within the study area. Understanding how commuters respond to extreme heat or cold temperatures could provide further context to myopic choice of bicycling.

Finally, while the majority of this discussion focuses on the interpretation of the parameters within the myopic odds function, the policy implications of this model are also relevant. When examining the model at a high level context, the model findings and analysis from above is in agreement with the literature on biking behaviour, and particularly with the concept of bicycling culture. By examining the aggregate/population probability of reliance on a given mode, or furthermore, examining the heat maps (figures 3 and 4), it is possible to gain greater insight into some of the factors influencing biking culture relative to examining more conventional models or mode split statistics. The model provides an explanation as to how environmental and personal attributes define the alternatives that are considered by a decision-maker. While this model structure does not capture habitual travel patterns, which are also a large component of bicycling culture; the model does provide insight into part of the question of how to improve bicycling culture within an urban region. The next section of this analysis will apply this concept of 
inferring biking culture from the myopic odds function and PLC model structure to an interesting policy application.

\section{Policy exercise}

In order to further investigate the effect of the built environment and the associated policy implications on individuals' mode choice behaviour and myopic modal choice behaviour a policy analysis scenario was proposed. This analysis aimed to test the percent change of the bicycle mode share due to improvements in the bicycle infrastructure across Toronto. Of particular interest was the impact of expanding the existing bicycle infrastructure such that all individuals with less than $50 \%$ of their route to work covered by bike infrastructure would have improved coverage. Table 2 shows the change of mode choice probabilities and Table 3 shows the change in myopic choice probabilities for commuters according to the proposed change to the bicycle infrastructure. These changes in mode share and myopic probability consider individuals who have bicycle as a feasible mode of travel in their choice sets and less than $50 \%$ of their commuting route marked as bicycle lanes.

From a feasibility standpoint this equates to each commuter obtaining, on average, an additional 1.85 kilometers of bicycling infrastructure on his or her route to work. An individual's bicycle route will likely be unique, based on his or her home and work location. However some sections of these individuals' routes overlap with other commuters' paths. The exact length of bicycle infrastructure investment required to obtain the new mode shares involves a comprehensive spatial analysis and more specifically infrastructure investment optimization. A brief review of the literature on 
optimal bike infrastructure investment was found (Duthie and Unnikrishnan 2014), and it is possible that some of these techniques could be applied to determine the optimal location of bicycle infrastructure investment.

It is interesting to note that an increase in bike mode share in these policy scenarios is gained by large part at the expense of transit mode share. So, it can be inferred that in a competitive modal arrangement, increasing bike infrastructure may pose a better context for biking and thereby may cause a reduction in transit ridership.

\section{Conclusion and future work}

This paper applies an advanced econometric modelling approach to examine mode choice and modal dependence/ myopic choices of commuters who work and reside in dense urban metropolitan areas. The empirical model is specified to provide a better understanding of modal culture based on the concept of personal reactions to the built environment. This novel application of the model structure provides new insights into the magnitude of different variables influence on promoting bicycling culture in particular. This understanding provides policy and decision-makers with a tool to test different policies or strategies to encourage biking mode share. In particular, the application of this model to the bicycle infrastructure investment policy provided meaningful insights into how infrastructure investment affects overall modal share within the region. While this analysis provides a good first step in understanding bicycling behaviour, myopic choice 
and culture, there is certainly room for further investigation into these phenomenon, particularly in the context of choice set generation and policy implications.

While the PLC model applied within this paper provides valuable insights into both biking behaviour, and more directly, bicycling culture, there are other probabilistic model formulations that could also potentially be investigated. One such model structure is the independent availability model, which may further explain the concept of culture as forces that enable or block certain alternatives. Further to this, the TTS dataset used for the model estimation was limited with a number of personal variables, which may have a high degree of significance relative to modal reliance and culture. These include variables such as income, bicycle ownership, cultural background or time spent living in Canada. Finally, a more comprehensive examination of the costs and optimization techniques associated with improving bicycle infrastructure as a method for improving bicycle choice, mode share and bicycling culture within the region should be undertaken.

\section{Acknowledgement}

The research was funded by an NSERC Discovery Grant and an Early Research Award from MEDI. However, the views and opinions presented in the paper belong only to the authors.

\section{References}

Aldred, R. and Jungnickel, K. 2014. Why culture matters for transport policy: The case of cycling in the UK. Journal of Transport Geography 34: 78-87. 
Aptech Systems. 2014. GAUSS Engine and MAXLIK. http://www.aptech.com/ (accessed in Jan 2016)

Bordley, Robert F. 1990. "The Dogit Model is Applicable Even without Perfectly Captive Buyers." Transportation Research Part B: Methodological 24 (4): 315-323.

Buehler, Ralph and John Pucher. 2012. "Cycling to Work in 90 Large American Cities: New Evidence on the Role of Bike Paths and Lanes." Transportation 39 (2): 409432.

Chu, You-Lian. 2010. "A Combined Destination and Route Choice Model for Capturing both Compulsory and Discretionary Trips.".

Chu, You-Lian 2009. "Work Departure Time Analysis using Dogit Ordered Generalized Extreme Value Model." Transportation Research Record: Journal of the Transportation Research Board 2132 (1): 42-49.

Data Management Group (DMG). 2013. 2011 Transportation Tomorrow Survey: Data Management Group.

Dill, J. and T. Carr. 2003. "Bicycle Commuting and Facilities in Major U.S. Cities: If You Build them, Commuters Will use Them." Transportation Research Record (1828): 116.

Duthie, Jennifer and Avinash Unnikrishnan. 2014. "Optimization Framework for Bicycle Network Design." Journal of Transportation Engineering 140 (7): 4014028.

Fry, Tim RL and Mark N. Harris. 2005. "The Dogit Ordered Generalized Extreme Value Model." Australian \& New Zealand Journal of Statistics 47 (4): 531-542.

Gaudry, Marc JI. 1980. "Dogit and Logit Models of Travel Mode Choice in Montreal." Canadian Journal of Economics 13 (2): 268-279.

Gaundry, Marc JI and Marcel G. Dagenais. 1979. "The Dogit Model." Transportation Research Part B: Methodological 13 (2): 105-111.

Habib, Khandker Nurul, Jenessa Mann, Mohamed Mahmoud, and Adam Weiss. 2014. "Synopsis of Bicycle Demand in the City of Toronto: Investigating the Effects of Perception, Consciousness and Comfortability on the Purpose of Biking and Bike Ownership." Transportation Research Part A: Policy and Practice 70: 67-80.

Habib, Khandker Nurul and Adam Weiss. 2014. "Evolution of Latent Modal Captivity and Mode Choice Patterns for Commuting Trips: A Longitudinal Analysis using Repeated Cross-Sectional Datasets." Transportation Research Part A: Policy and Practice 66: 39-51. 
Jacques, Cynthia, Kevin Manaugh, and Ahmed M. El-Geneidy. 2013. "Rescuing the Captive [Mode] User: An Alternative Approach to Transport Market Segmentation." Transportation 40 (3): 625-645.

Manski, Charles F. 1977. "The Structure of Random Utility Models." Theory and Decision 8 (3): 229-254.

McCarthy, Patrick S. 1997. "The Role of Captivity in Aggregate Share Models of Intercity Passenger Travel." Journal of Transport Economics and Policy 31 (3): 293308.

Metrolinx. 2008. The Big Move: Transforming Transportation in the Greater Toronto and Hamilton Area (GTHA).

Pucher, J. and R. Buehler. 2006. "Why Canadians Cycle More than Americans: A Comparative Analysis of Bicycling Trends and Policies." Transport Policy 13 (3): 265-279.

Statistics Canada. "Toronto, Ontario (Code 535) and Ontario (Code 35) (Table). Census Profile. 2011 Census.", accessed 07/13, 2014, http://www12.statcan.gc.ca/censusrecensement/2011/dp-pd/prof/index.cfm?Lang=E.

Stopher, Peter R. 1980. "Captivity and Choice in Travel-Behavior Models." Transportation Engineering Journal of the American Society of Civil Engineers 106 (4): 427-435.

Swait, Joffre. 1984. "Probabilistic Choice Set Formation in Transportation Demand Models." Ph.D, Massachusetts Institute of Technology.

Swait, Joffre and Moshe Ben-Akiva. 1986. "Analysis of the Effects of Captivity on Travel Time and Cost Elasticities." Behavioural Research for Transport Policy: 119134.

Swait, Joffre and Moshe Ben-Akiva 1987a. "Empirical Test of a Constrained Choice Discrete Model: Mode Choice in Sao Paulo, Brazil." Transportation Research Part B: Methodological 21 (2): 103-115.

Swait, Joffre and Moshe Ben-Akiva 1987b. "Incorporating Random Constraints in Discrete Models of Choice Set Generation." Transportation Research Part B: Methodological 21 (2): 91-102.

Tardiff, Timothy J. 1976. "Perception of the Availability of Transportation Alternatives for various Trip Purposes." Transportation Research Record: Journal of the Transportation Research Board 592: 12-16. 
Train, Kenneth. 2009. Discrete Choice Methods with Simulation. Second Edition ed. 32 Avenue of the Americas, New York, NY 10013-2473, USA: Cambridge university press.

Tudela, A., Khandker M. Nurul Habib, and Ahmed O. Idris. 2011. "Tudela, A., Nurul Habib, K. M., \& Idris, A. O. (2011). Semantic Approach to Capture Psychological Factors Affecting Mode Choice: Comparative Results from Canada and Chile." Termas de Puyehue, Chile, . 


\section{TABLE 1 Empirical Model}

\begin{tabular}{|c|c|c|c|c|}
\hline Model & \multirow{2}{*}{\multicolumn{2}{|c|}{$\begin{array}{c}\text { MNL } \\
-5000.12\end{array}$}} & \multirow{2}{*}{\multicolumn{2}{|c|}{$\begin{array}{c}\text { PLC } \\
-494375\end{array}$}} \\
\hline Log likelihood of Full Model & & & & \\
\hline Log likelihood of Constant-Only Model & \multicolumn{2}{|c|}{-6392.84} & \multicolumn{2}{|c|}{-6364.41} \\
\hline Rho-square value & \multicolumn{2}{|c|}{0.218} & \multicolumn{2}{|c|}{0.223} \\
\hline Number of Observations & \multicolumn{2}{|c|}{6556.00} & \multicolumn{2}{|c|}{6556.00} \\
\hline Systematic Utility Function: & Parameter & $t$-Stat & Parameter & $t$-Stat \\
\hline \multicolumn{5}{|l|}{ Alternative Specific Constant } \\
\hline Auto Driver & 0.4148 & 5.083 & -1.7325 & -0.589 \\
\hline Auto Passenger & 0 & 0 & 0 & 0 \\
\hline Local Transit Walk Access & 2.2828 & 13.157 & 12.6321 & 5.76 \\
\hline Bike & -0.0716 & -0.255 & -8.329 & -0.04 \\
\hline Walk & 2.8812 & 14.814 & 2.8549 & 2.576 \\
\hline Travel Cost/Fare (CDN \$) & -0.0386 & -10.31 & -0.1921 & -4.931 \\
\hline In Vehicle Travel Time (minutes) & -0.2401 & -5.358 & -4.6379 & -5.314 \\
\hline Wait Time (minutes) & -0.1295 & -5.708 & -0.6534 & -4.643 \\
\hline Access, Egress and Transfer Times (minutes) & -0.0355 & -4.938 & -0.166 & -4.359 \\
\hline \multicolumn{5}{|c|}{ Parameterized Captivity Function (for MNL these variables go into the systematic utility function) } \\
\hline \multicolumn{5}{|l|}{ Constant } \\
\hline Auto Driver & --- & --- & -0.5191 & -4.499 \\
\hline Auto Passenger & --- & --- & -1.3416 & -7.844 \\
\hline Local Transit Walk Access & --- & --- & -0.1981 & -0.818 \\
\hline Bike & --- & --- & -0.9725 & -3.221 \\
\hline Walk & --- & --- & 1.045 & 5.042 \\
\hline \multicolumn{5}{|l|}{ Auto Driver } \\
\hline Number of Vehicles $>1$ & 1.0194 & 13.416 & 0.9134 & 11.644 \\
\hline Free Parking at Work & 1.3977 & 17.274 & -0.0328 & -6.705 \\
\hline \multicolumn{5}{|l|}{ Local Transit Walk Access } \\
\hline Transit Pass & 2.5721 & 30.711 & 3.3967 & 17.59 \\
\hline Ratio of Number of Vehicles to Number of Persons & -0.0761 & -3.537 & -1.2597 & -5.589 \\
\hline \multicolumn{5}{|l|}{ Bike } \\
\hline Age $>60$ & 0.4157 & 3.421 & -0.598 & -3.32 \\
\hline Temperature $>10\left({ }^{\circ} \mathrm{C}\right)$ & 0.1013 & 2.335 & 0.3932 & 3.238 \\
\hline $\ln \%$ of path covered with bike infrastructure & -0.0311 & -6.468 & 0.1086 & 2.487 \\
\hline Number of Intersections & 0.4873 & 2.722 & -0.0328 & -6.705 \\
\hline Work Location in Downtown (Planning District 1) & 0.4873 & 2.722 & 0.3675 & 2.041 \\
\hline Male & 1.1215 & 8.594 & 1.0913 & 7.988 \\
\hline Peak Period & -0.3252 & -2.406 & -0.3539 & -2.611 \\
\hline \multicolumn{5}{|l|}{ Walk } \\
\hline Age $>60$ & -0.5205 & -2.987 & -0.598 & -3.32 \\
\hline Home and Work Locations in the Same Planning District & 0.2728 & 1.574 & 0.3645 & 1.971 \\
\hline
\end{tabular}


Table 2 Change in mode share based on increase in cycling infrastructure for individuals with less than $50 \%$ of their path covered by cycling infrastructure and biking in their choice set

\begin{tabular}{|c|c|c|c|c|c|}
\hline $\begin{array}{c}\text { Scenario } \mid \\
\text { Alternative }\end{array}$ & $\begin{array}{c}\text { Automobile } \\
\text { Driver }\end{array}$ & $\begin{array}{c}\text { Automobile } \\
\text { Passenger }\end{array}$ & $\begin{array}{c}\text { Local Transit } \\
\text { Rider }\end{array}$ & Cyclist & Walking \\
\hline Base Case & $19.96 \%$ & $9.93 \%$ & $46.05 \%$ & $11.11 \%$ & $12.95 \%$ \\
\hline $\begin{array}{c}\text { 50\% Cycling } \\
\text { Infrastructure }\end{array}$ & $19.70 \%$ & $9.71 \%$ & $45.54 \%$ & $12.23 \%$ & $12.82 \%$ \\
\hline $\begin{array}{c}\text { 90\% Cycling } \\
\text { Infrastructure }\end{array}$ & $19.56 \%$ & $9.59 \%$ & $45.26 \%$ & $12.83 \%$ & $12.76 \%$ \\
\hline
\end{tabular}


Table 3 Change in myopic probability based on increase in cycling infrastructure for individuals with less than $\mathbf{5 0 \%}$ of their path covered by cycling infrastructure and biking in their choice set

\begin{tabular}{|c|c|c|c|c|c|c|}
\hline $\begin{array}{c}\text { Scenario } \\
\text { Alternative }\end{array}$ & Rational & $\begin{array}{c}\text { Automobile } \\
\text { driver }\end{array}$ & $\begin{array}{c}\text { Automobile } \\
\text { Passenger }\end{array}$ & $\begin{array}{c}\text { Local } \\
\text { Transit } \\
\text { Rider }\end{array}$ & Cyclist & Walking \\
\hline Base Case & $21.93 \%$ & $16.31 \%$ & $57.30 \%$ & $36.28 \%$ & $85.90 \%$ & $11.15 \%$ \\
\hline $\begin{array}{c}\text { 50\% Cycling } \\
\text { Infrastructure }\end{array}$ & $21.60 \%$ & $16.14 \%$ & $56.50 \%$ & $36.05 \%$ & $95.00 \%$ & $11.06 \%$ \\
\hline $\begin{array}{c}\text { 90\% Cycling } \\
\text { Infrastructure }\end{array}$ & $21.42 \%$ & $16.05 \%$ & $56.00 \%$ & $35.92 \%$ & $10.00 \%$ & $11.01 \%$ \\
\hline
\end{tabular}


Figure 1: Modal Shares

Figure 2: Transit and Bike Infrastructure

Figure 3: Reliance on Private Automobile

Figure 4: Reliance on Bicycle

https://mc06.manuscriptcentral.com/cjce-pubs 


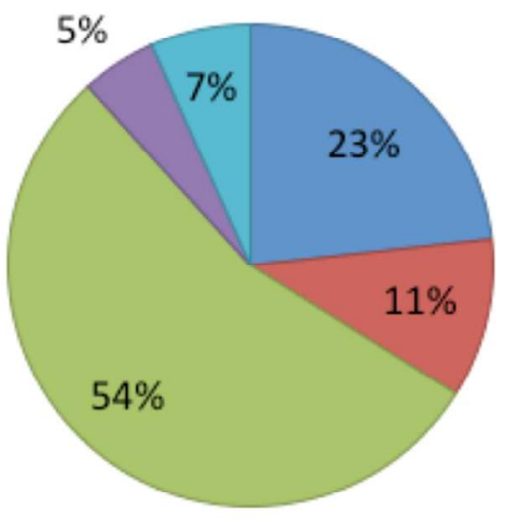

$\square$ Auto Driver

$\square$ Auto Passenger

$\square$ Transit

$\square$ Bike

$\square$ Walk 


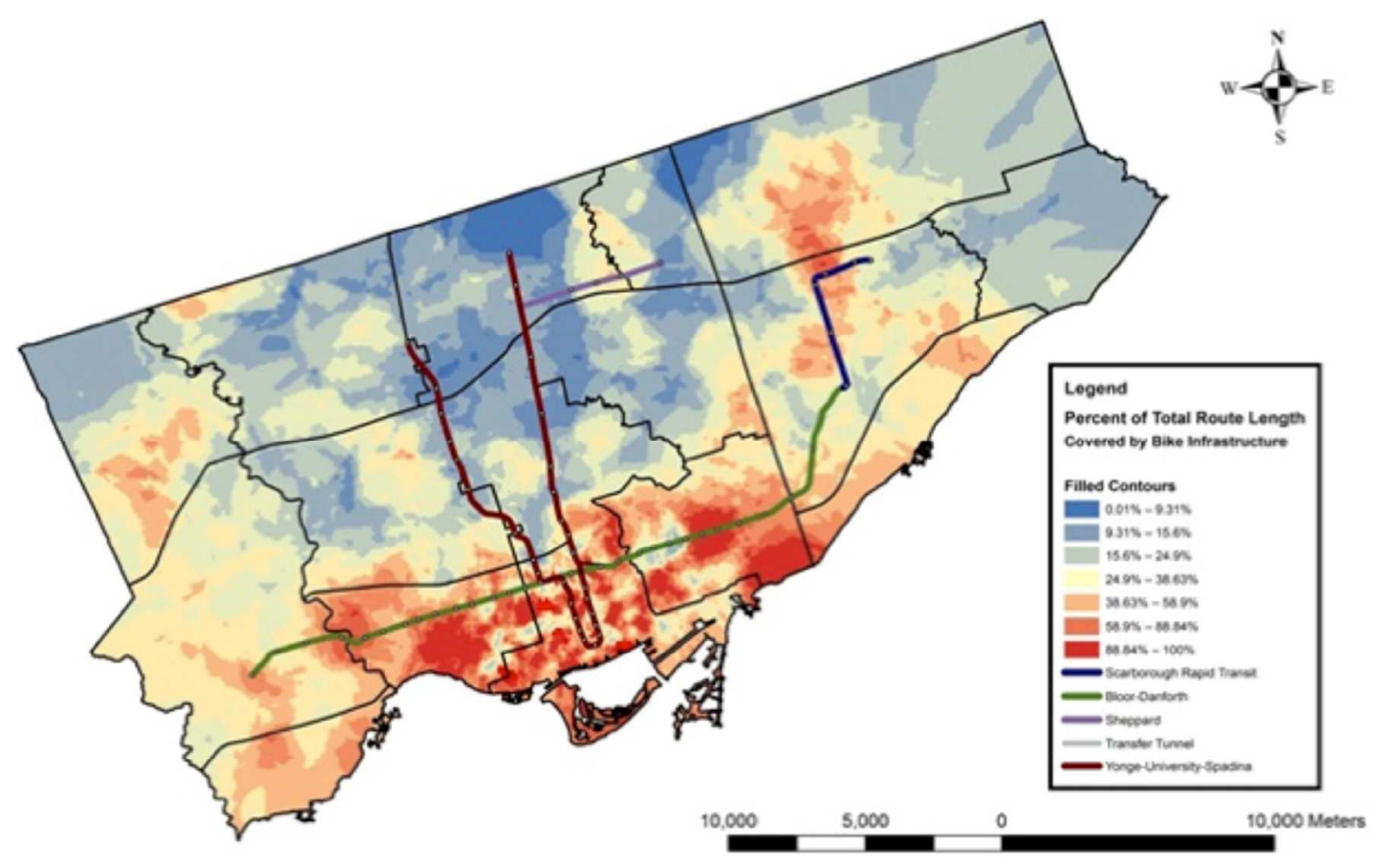




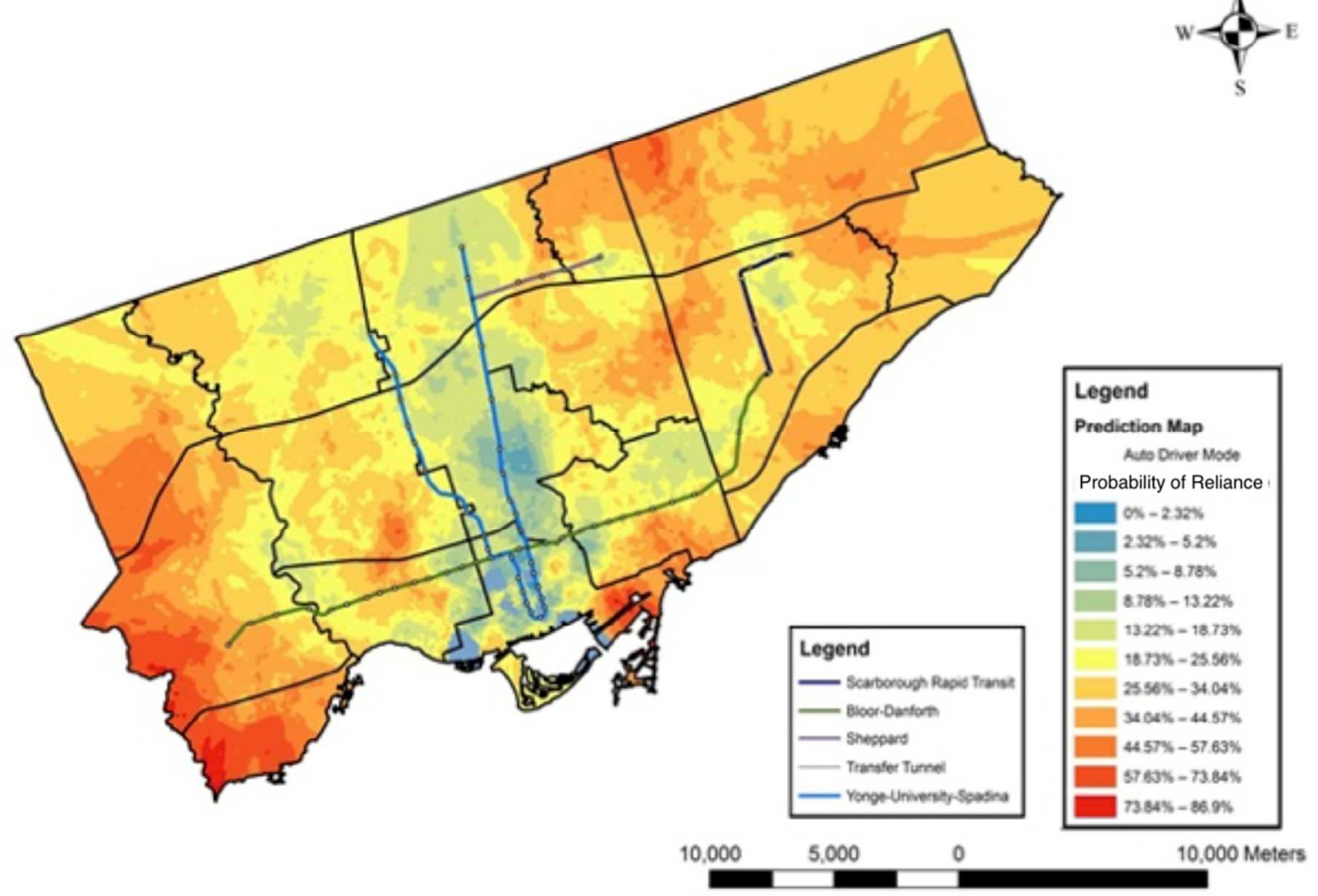




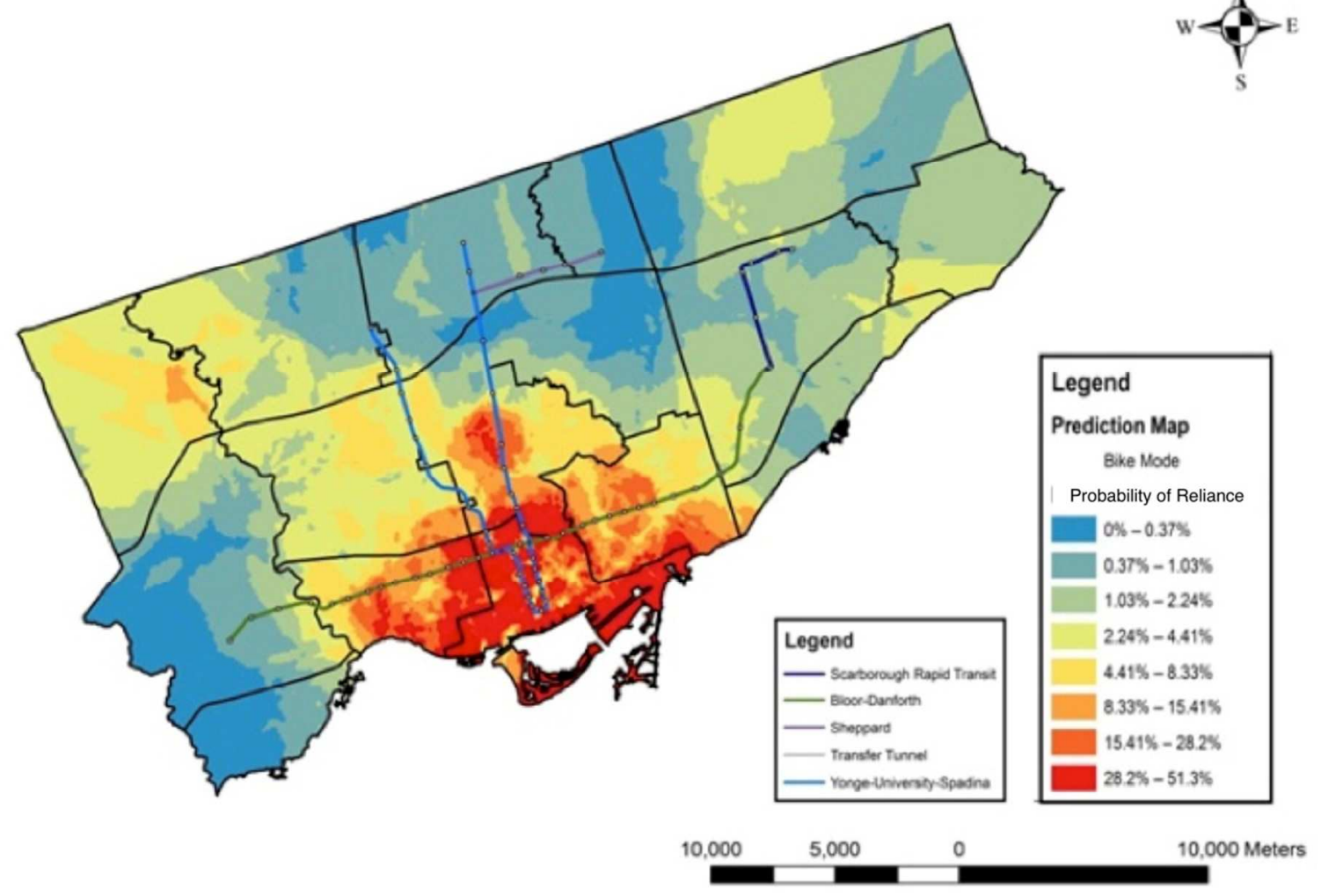

